Bryn Mawr College

Scholarship, Research, and Creative Work at Bryn Mawr

College

2007

\title{
Asymmetric Domain Nucleation and Unusual Magnetization Reversal in Ultrathin Co Films with Perpendicular Anisotropy
}

Y.L. Iunin

Y.P. Kabanov

V. I. Nikitenko

Xuemei Cheng

Bryn Mawr College, xcheng@brynmawr.edu

D. Clarke

See next page for additional authors

Let us know how access to this document benefits you.

Follow this and additional works at: http://repository.brynmawr.edu/physics_pubs

Part of the Physics Commons

\section{Custom Citation}

Y. L. Iunin et al., Phys. Rev. Lett. 98, 117204 (2007).

This paper is posted at Scholarship, Research, and Creative Work at Bryn Mawr College. http://repository.brynmawr.edu/physics_pubs/62 
Authors

Y. L. Iunin, Y. P. Kabanov, V. I. Nikitenko, Xuemei Cheng, D. Clarke, O. A. Tretiakov, O. Tchernyshyov, A. J. Shapiro, R. D. Shull, and C. L. Chien 


\title{
Asymmetric Domain Nucleation and Unusual Magnetization Reversal in Ultrathin Co Films with Perpendicular Anisotropy
}

\author{
Y.L. Iunin, ${ }^{1}$ Y. P. Kabanov, ${ }^{1}$ V. I. Nikitenko,,${ }^{1,2,3}$ X. M. Cheng, ${ }^{2}$ D. Clarke, ${ }^{2}$ O. A. Tretiakov, ${ }^{2}$ O. Tchernyshyov, ${ }^{2}$ \\ A. J. Shapiro, ${ }^{3}$ R. D. Shull, ${ }^{2}$ and C. L. Chien ${ }^{2}$ \\ ${ }^{1}$ Institute of Solid State Physics, Russian Academy of Sciences, Chernogolovka 142432, Russia \\ ${ }^{2}$ The Johns Hopkins University, Baltimore 21218, Maryland, USA \\ ${ }^{3}$ National Institute of Standards and Technology, Gaithersburg 20899, Maryland, USA
}

(Received 15 June 2006; published 16 March 2007)

\begin{abstract}
We report unexpected phenomena during magnetization reversal in ultrathin Co films and $\mathrm{Co} / \mathrm{Pt}$ multilayers with perpendicular anisotropy. Using magneto-optical Kerr microscopy and magnetic force microscopy we have observed asymmetrical nucleation centers where the reversal begins for one direction of the field only and is characterized by an acute asymmetry of domain-wall mobility. We have also observed magnetic domains with a continuously varying average magnetization, which can be explained in terms of the coexistence of three magnetic phases: up, down, and striped.
\end{abstract}

DOI: 10.1103/PhysRevLett.98.117204

PACS numbers: 75.60.Ch, 75.70.Ak

Ultrathin ferromagnetic films are quasi-twodimensional magnets possessing fascinating physical properties [1]. They have strong perpendicular magnetic anisotropy (PMA) exceeding the influence of the demagnetizing field. Ultrathin films with PMA are a unique medium for exploring magnetic phenomena of fundamental importance, such as magnetization reversal and magnetoresistance, which are essential for technological applications of high-density perpendicular magnetic recording. The strong PMA in materials such as $[\mathrm{Co} / \mathrm{Pt}]_{n}$ multilayers, where $n$ is the number of repeats, is the result of a strong interfacial surface anisotropy, which is inversely proportional to the thickness of the ferromagnetic layer. As the film thickness increases, the magnetization returns to the plane of the film.

The dynamics of magnetization reversal in multilayers with PMA is determined by two fundamental processes: the nucleation of domains with reversed magnetization and the growth of such domains mediated by the motion of domain walls (DWs). These processes have been studied in detail in ultrathin Co films [2-8]. It was shown that, regardless of the field direction, round-shaped reversed domains nucleate at the same positions and grow isotropically until they fill the entire sample. Domain-wall velocities in ultrathin Co films were measured for a wide range of magnetic fields and temperature. The DW motion was found to be either thermally activated or viscous depending upon the strength of the magnetic field [3].

The magnetization reversal in $[\mathrm{Co} / \mathrm{Pt}]_{n}$ multilayers consisting of many repeats differs drastically [9-17] from that of ultrathin Co films. The reversal in multilayers with a large $n$ (e.g., $n=50$ [14]) also begins by the nucleation of small bubble domains. However, in contrast to ultrathin films, these domains grow into one-dimensional lines forming orientationally disordered arrays of stripes where the magnetization points alternatively up and down. Changes in magnetization upon application of an external magnetic field are caused by changes in the widths of the up and down stripes. These multilayers often demonstrate dendritelike domain patterns $[10,13]$.

The aim of this work is to study in detail the nucleation and motion of DWs in Co ultrathin films with PMA and to reveal the manner in which the number of Co layers $n$ in $[\mathrm{Co} / \mathrm{Pt}]_{n}(n=1,2,4,16)$ multilayers affects the nucleation and growth of the magnetic domains. We report the observation of hitherto unknown asymmetrical magnetic domain (MD) nucleation centers in ultrathin structures. The new MDs nucleate at different positions for magnetization M pointing up and down. We have also found a pronounced asymmetry in the mobility of the DWs, which is in sharp contrast to the expected symmetry of magnetic reversal in general. We have also observed an unusual phenomenon of magnetic domains with a fixed boundary but continuously varying magnetization.

$\operatorname{Pt}(100 \AA) / \operatorname{Co}(d) / \operatorname{Pt}(30 \AA) \quad$ films and $\operatorname{Pt}(100 \AA) /$ $[\mathrm{Co}(d) / \operatorname{Pt}(10 \AA)]_{n} / \operatorname{Pt}(20 \AA)$ multilayers $(d=4,6,8 \AA$, $n=2,4,16)$ were grown by dc magnetron sputtering onto Si (111) substrates. The domain structure evolution was investigated in real time by magneto-optical Kerr effect (MOKE) microscopy at various magnetic fields, applied perpendicular to the film plane, and by magnetic force microscopy (MFM) imaging of samples previously magnetized to the desired magnetic states by an electromagnet.

Average magnetization as a function of the field was determined by Hall resistance measurements with the applied magnetic field $\mathbf{H}$ perpendicular to the film plane. Since the extraordinary Hall resistance $R_{H}$ in multilayers with PMA is proportional to $M$, the field dependence of $R_{H}$ yields the curve $M(H)$. The hysteresis loops of $[\mathrm{Co} / \mathrm{Pt}]_{n}$ samples with $n=1,2$, and 4 [18] were highly square, whereas $[\mathrm{Co} / \mathrm{Pt}]_{16}$ had a slanted loop similar to that measured in Refs. $[11,12,14,16]$. The coercivity $H_{C}$ also increases with the number of Co layers. To study the nucleation and evolution of the domains the field was 
gradually reduced from $\mu_{0} H=60 \mathrm{mT}$, far exceeding the sample coercivity, to a point where magnetic domains began to nucleate and grow.

Figure 1 shows the nucleation and evolution of MDs in $[\mathrm{Co} / \mathrm{Pt}]_{2}$ in magnetic fields of both signs. One can see the usual "symmetrical" ( $S$-type) nucleation centers where bubble domains form, regardless of the field sign. Reversing the magnetic field before the domain wall reached the sample boundaries led to the formation of a ring structure, as shown in Ref. [7] thanks to the formation of a new domain at the same nucleation center. Most unusually, one also sees "asymmetrical" nucleation centers producing MDs of only one magnetization direction, either downward (type $A_{\text {down }}$ ) or upward (type $A_{\text {up }}$ ), but not both, as indicated in Fig. 1 by the arrows. After the magnetic field has been reversed, the domains of types $A_{\text {up }}$ and $A_{\text {down }}$ switch from growing to shrinking but no new domain forms at the nucleation center. We observed the asymmetry of domain nucleation in $[\mathrm{Co} / \mathrm{Pt}]_{n}$ multilayers with $n=1$, 2 , and 4. Asymmetric nucleation centers were reliably suppressed after the applied field was increased to $\mu_{0} H=$ $120 \mathrm{mT}$. However, they reappeared in the next cycle.

To further study the characteristics of symmetric and asymmetric domain evolution, we have measured the DW mobility. To that end, the magnetic field was kept constant after the MD formation and the displacement of the DW was measured as a function of time. The results of the measurements of $S$-type DWs are shown in Fig. 2(a). The
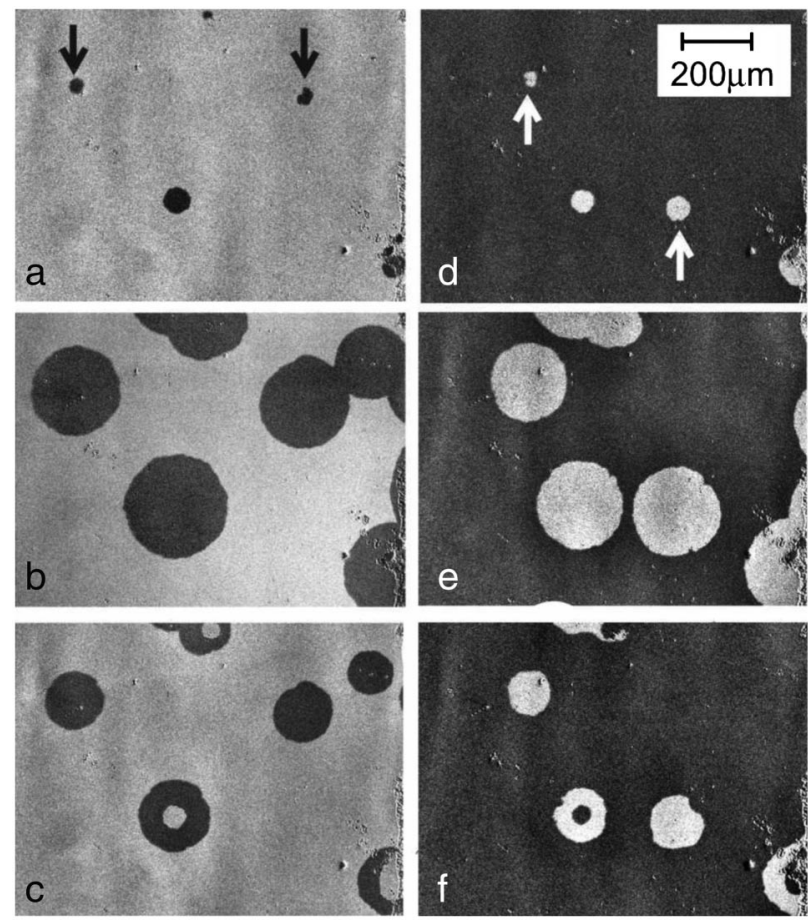

FIG. 1. MOKE images of the evolution of the $[\mathrm{Co} / \mathrm{Pt}]_{2}$ domain structure under magnetic fields $\mu_{0} H=-17.3 \mathrm{mT}(\mathrm{a}, \mathrm{b})$, $\mu_{0} H=+17.3 \mathrm{mT}(\mathrm{d}),(\mathrm{e})$, and after magnetic field cycling (c),(f); arrows indicate the domains produced by asymmetric centers.
DW displacement is linear in time, with a well-defined DW velocity. The speed of symmetrical DW is the same for either direction of the field.

In contrast, the $A$-type DW backward motion is significantly faster than that of DW forward motion attained in a field of the same strength and opposite direction. In fact, at $\mu_{0} H= \pm 1.52 \mathrm{mT}$, the average DW velocity during DW backward motion is about twice as large as that during DW forward motion [Fig. 2(b)]. The larger the magnetic field the less the asymmetry in DW mobility. No noticeable asymmetry of DW mobility was observed in a single-layer Co film with higher coercivity $\mu_{0} H_{c}=8.8 \mathrm{mT}$ and in the $[\mathrm{Co} / \mathrm{Pt}]_{2}$ sample $\left(\mu_{0} H_{c}=19 \mathrm{mT}\right)$.

Increase in the number of Co layers has resulted in one more unexpected outcome. In $[\mathrm{Co} / \mathrm{Pt}]_{4}$, MDs nucleate and spread by DW motion [Fig. 3(a) and 3(b)] similar to those in single-layer Co films and the $[\mathrm{Co} / \mathrm{Pt}]_{2}$ sample. However, after the magnetic field has been reversed, the MDs do not reduce their size as they do in single-layer Co films and the $[\mathrm{Co} / \mathrm{Pt}]_{2}$ sample. Instead, the reversal is accompanied by a gradual fading of the MOKE contrast [Figs. 3(c) and 3(d)] before disappearing. MFM imaging clearly shows that the magnetization in the newly nucleated domain is not uniform but contains stripes of opposite magnetization [18]. Evidently, the decrease in magneto-optical contrast [Figs. 3(c) and 3(d)] corresponds to the change in the widths of the up and down stripes.

As is already known, increasing the number of repeats (e.g., $[\mathrm{Co} / \mathrm{Pt}]_{16}$ ) results in a qualitative change in the magnetization reversal mechanism. After nucleation, the small bubble domains become elongated and eventually a dendritic structure forms similar to those in Ref. [10]. The dendrites grow until they cover the entire surface of the sample. The width of the reversed domains remains relatively constant throughout the growth in the dendritic structure. The channels then slowly expand once the dendritic structure covers the entire sample [13]. These two growth stages of the domain structure are responsible for the slanted shape of the hysteresis loop. The three unusual

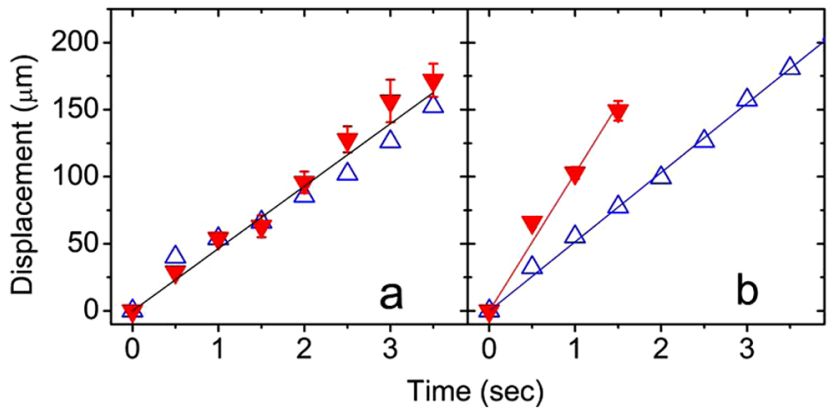

FIG. 2 (color online). Time dependence of DW displacements for a single Co layer film with coercivity of $\mu_{0} H_{c}=2.3 \mathrm{mT}$ under magnetic field cycling $\mu_{0} H= \pm 1.52 \mathrm{mT}$ of domains produced by (a) symmetric (type $\mathrm{S}$ ) center and (b) asymmetric (type $A_{\text {up }}$ ) center; both DW forward motion $(\triangle)$ and DW backward motion $(\boldsymbol{\nabla})$ data are indicated. 

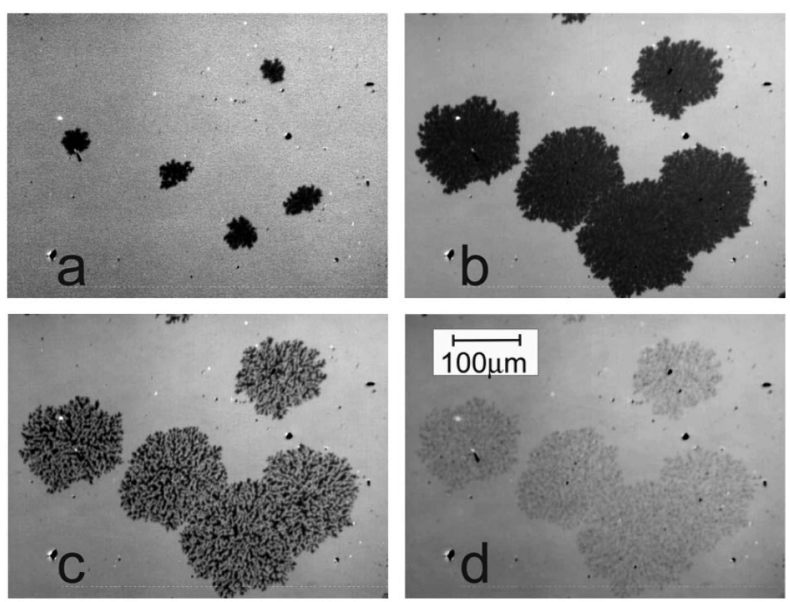

FIG. 3. MOKE images of the evolution of the $[\mathrm{Co} / \mathrm{Pt}]_{4}$ domain structure with $\mu_{0} H=+23.4 \mathrm{mT}$ (a),(b), and after a magnetic field switch to $\mu_{0} H=-23.4 \mathrm{mT}$ (c),(d).

effects revealed in this work are the asymmetry of the activity of nucleation centers, asymmetry of the DW mobility, and domains with fading MOKE contrast. In addition, there is the well-established but unexplained dependence on the number of repeats $n$ in $[\mathrm{Co} / \mathrm{Pt}]_{n}$ multilayers. A theoretical model described in the following accounts well for the features of fading domains and dependence on the number of repeats. We also suggest possible causes of the asymmetric DW nucleation and motion.

Observation of labyrinthlike domains in $[\mathrm{Co} / \mathrm{Pt}]_{4}$ and $[\mathrm{Co} / \mathrm{Pt}]_{16}$ points to the presence of a stripe phase in a twodimensional ferromagnet [19]. This phase can be viewed as the coexistence of elongated domains of up and down magnetization on a mesoscopic length scale caused by a competition between the spin-orbit, exchange, and dipolar interactions [20]. The stripes readily lose translational and orientational order as a result of thermal fluctuations $[21,22]$. However, their average period can be determined within a mean-field approach. Assuming that the magnetization lies in the $y z$ plane ( $z$ being the out-of-plane coordinate) and varies along the $x$ direction only, $\mathbf{M}(x)=$ $(0, M \cos \theta(x), M \sin \theta(x))$, we obtain the energy density [21]

$$
\begin{aligned}
\frac{E}{w t}= & \int d x\left(A \theta^{\prime 2}-K \cos ^{2} \theta-\mu_{0} H M \cos \theta\right) \\
& +\frac{\mu_{0} M^{2} t}{4 \pi} \int d x \int d x^{\prime} \frac{\cos \theta(x) \cos \theta\left(x^{\prime}\right)}{\left(x-x^{\prime}\right)^{2}+(t / 2)^{2}} .
\end{aligned}
$$

Here $w$ and $t$ are the width and thickness of the magnetic film, $A \approx 1.3 \times 10^{-11} \mathrm{~J} / \mathrm{m}$ is the exchange constant (the value accepted for bulk Co), $M \approx 1.0 \times 10^{-6} \mathrm{~A} / \mathrm{m}$ is the saturation magnetization, and $K$ is the easy-axis anisotropy constant. The stripes are observable only if $K$ is close to the critical value $K_{0}=\mu_{0} M^{2} / 2$ [20]. If the anisotropy is too strong, the stripe period, which varies exponentially with $K-K_{0}$, becomes very large. If it is too weak, the magnetization lies in the plane of the film.
A phase diagram depicting the types of domain phases observed with variations in anisotropy and field can be constructed as discussed by Berger and Erickson [23]. Here we outline the general results of such a phase diagram [24]. We pay particular attention to the metastable states, as they are important for understanding the magnetization reversal. The phase diagram in the $(K, H)$ plane has a universal shape if one uses dimensionless anisotropy $\kappa=\left(K-K_{0}\right) / K_{0}$ and field $h=H / M$. This is shown in Fig. 4. A characteristic scale of these quantities is set by the film thickness: $\kappa_{0}=h_{0}=(t / 4 \lambda)^{2}$, where $\lambda=$ $\left(2 A / \mu_{0} M^{2}\right)^{1 / 2} \approx 5 \mathrm{~nm}$ is the magnetic exchange length. In addition to regions containing single phases and separated by continuous phase transitions (solid lines), there are also regions of coexistence where one or more phases are metastable. The metastable states decay along dashed lines. The limits of metastability of the striped phases (short-dashed line) are determined by a variational method employing a single-harmonic approximation $\cos \theta(x)=$ $\sigma_{0}+\sigma_{1} \cos k x$. The rest of the boundaries are exact.

The behavior of $[\mathrm{Co} / \mathrm{Pt}]_{4}$ can be explained if we assign to it a small positive $\kappa$ close to the intersection of the metastability boundaries of the uniform and striped phases (Fig. 4). Starting with the uniform up state in a positive field (point 1) and reducing the field to a negative value we come to point 2 where the up state decays into the stripe phase. Because of inhomogeneities present in the sample, the striped phase will first appear at a few isolated spots with the rest of the sample remaining in the up state. If now the field is gradually ramped back to positive values $(2 \rightarrow 3)$, the average magnetization within the striped spots will increase continuously as the up stripes become wider and the down stripes narrower, in accordance with observations.

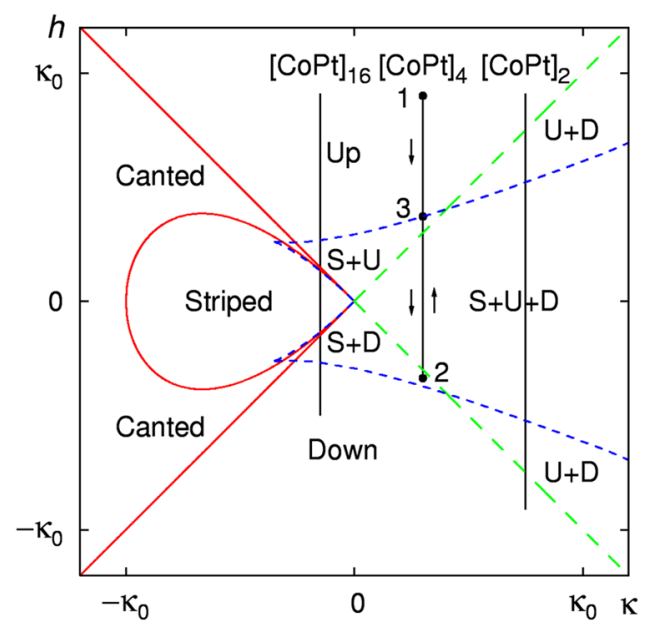

FIG. 4 (color online). Phases of a magnetic film with PMA as a function of dimensionless anisotropy $\kappa=\left(K-K_{0}\right) / K_{0}$ and field $h=H / M$. Continuous phase transitions are shown as solid lines, limits of metastability as dashed lines. Regions containing striped (S), upward magnetized (U), and downward magnetized (D) domains are shown. 
The proposed location of the $[\mathrm{Co} / \mathrm{Pt}]_{4}$ line in the phase diagram is confirmed by an estimate of the dimensionless anisotropy. Since the reversal begins at a negative field $\mu_{0} H=-19.0 \mathrm{mT}$, we obtain $\kappa=-H / M=0.015$ that is positive and small on the scale of $k_{0}=(t / 4 \lambda)^{2}=0.10$. Approximate locations of the other two samples are also indicated by faint vertical lines in Fig. 4. The thinner $[\mathrm{Co} / \mathrm{Pt}]_{2}$ has a smaller scale $\kappa_{0}$ and, assuming the same anisotropy $\kappa$, is expected to have a larger ratio $\kappa / \kappa_{0}$. In that case the reversal begins in a large field $H$ where the stripe phase no longer exists; the up and down states decay directly into each other yielding square magnetization loops. On the other hand, the thickest sample $[\mathrm{Co} / \mathrm{Pt}]_{16}$ should have a small $\kappa / \kappa_{0}$. Its peculiar hysteresis curve is consistent with a small negative $\kappa / \kappa_{0}$. The change of sign of the parameter $\kappa=\left(K-K_{0}\right) / K_{0}$ likely results from small systematic variations of the anisotropy $K$ with the overall thickness of the sample.

Let us also briefly mention possible causes of the unusual asymmetrical effects: activity of nucleation centers and DW mobility. It can be caused by the presence of islands with very high coercivity and opposite magnetization directions as was observed by the spin-polarized scanning tunneling microscopy [25]. Another potential cause for the formation of local unidirectional anisotropy is the exchange coupling between islands with ferromagnetic and antiferromagnetic (AF) order [26-30]. The AF ordering may occur due to oxidation of some Co islands and formation of $\mathrm{CoO}$ [26], at the interface steps [27], or at the Co surface like in an Fe/W(001) ultrathin film [30]. These potential causes were not supported by further experiments. For instance, we were able to fully suppress the asymmetric nucleation by ramping up the field to $\mu_{0} H=$ $120 \mathrm{mT}$. This rather modest field value rules out antiferromagnetic islands (e.g., $\mathrm{CoO}$ ): much higher fields are normally required to overcome the strength of exchange in an antiferromagnet. Furthermore, the asymmetric nucleation returned after the next reversal cycle with a moderate maximum field.

Alternatively, the asymmetric nucleation may be related to the chiral nature of the domain walls: the vector of magnetization points along the direction of the wall. As a result, a small down domain surrounded by a large up area has a topologically nontrivial configuration known as the Skyrmion [31]. If Skyrmions remain stable against collapse to the atomic scale [32], the down domains shrink to a small but finite radius in an upward field and reappear when the field is reversed. The domain walls may have different chiralities and also be composed of parts having opposite chiralities and separated by quasi-Bloch lines. These distinctions may be the cause of the difference in mobility of the DWs. One more reason for the asymmetry of DW mobility may be the jerky remagnetization of nanosized islands near the DW front. Further work is needed to confirm or rule out these scenarios.

In conclusion, we have observed the unusual formation of domains of reversed magnetization in $[\mathrm{Co} / \mathrm{Pt}]_{n}(n=1$,
2,4) with PMA. At some nucleation centers domains of opposite magnetization arise only for one direction of the reversing field but not for the other. The DWs formed by such nucleation centers in Co films move backward faster than they move forward in a field of the same strength. Samples with a larger number of Co layers, $[\mathrm{Co} / \mathrm{Pt}]_{4}$ and $[\mathrm{Co} / \mathrm{Pt}]_{16}$, reveal the presence of a stripe phase coexisting with the up and down polarized phases. The observed behavior is consistent with a simple mean-field model of the striped phase that will be detailed elsewhere [24].

This work was supported in part by the NSF Grants No. DMR-0403849 and No. DMR-0520491.

[1] B. Heinrich and J.F. Cochran, Adv. Phys. 42, 523 (1993).

[2] J. Pommier et al., Phys. Rev. Lett. 65, 2054 (1990).

[3] A. Kirilyuk et al., J. Magn. Magn. Mater. 171, 45 (1997).

[4] J. Ferre et al., Phys. Rev. B 55, 15092 (1997); Phys. Status Solidi A 201, 1386 (2004).

[5] U. Nowak et al., Phys. Rev. B 56, 8143 (1997).

[6] S. Lemerle et al., Phys. Rev. Lett. 80, 849 (1998).

[7] M. Kisielewski et al., J. Magn. Magn. Mater. 260, 231 (2003).

[8] L. Krusin-Elbaum et al., Nature (London) 410, 444 (2001).

[9] D. Weller et al., J. Appl. Phys. 89, 7525 (2001).

[10] S. B. Choe and S. C. Shin, Phys. Rev. B 57, 1085 (1998).

[11] C. L. Canedy, X. W. Li, and G. Xiao, Phys. Rev. B 62, 508 (2000).

[12] J.C. A. Huang et al., J. Magn. Magn. Mater. 209, 90 (2000).

[13] R. C. Woodward et al., J. Appl. Phys. 93, 6567 (2003).

[14] J. E. Davies et al., Phys. Rev. B 70, 224434 (2004).

[15] A. Schwarz et al., Phys. Rev. Lett. 92, 077206 (2004).

[16] R. A. Fry, L. H. Bennett, and E. Della Torre, J. Appl. Phys. 85, 5169 (1999).

[17] M. S. Pierce et al., Phys. Rev. Lett. 94, 017202 (2005).

[18] X. M. Cheng et al., J. Appl. Phys. 99, 08 C905 (2006).

[19] R. Allenspach and A. Bischof, Phys. Rev. Lett. 69, 3385 (1992).

[20] Y. Yafet and E. M. Gyorgy, Phys. Rev. B 38, 9145 (1988).

[21] A. B. Kashuba and V.L. Pokrovsky, Phys. Rev. B 48, 10335 (1993).

[22] A. Abanov et al., Phys. Rev. B 51, 1023 (1995).

[23] A. Berger and R. P. Erickson, J. Magn. Magn. Mater. 165, 70 (1997).

[24] D. Clarke, O. A. Tretiakov, and O. Tchernyshyov, condmat/0612346.

[25] O. Pietzsch et al., Phys. Rev. Lett. 92, 057202 (2004).

[26] W.H. Meiklejohn and C.P. Bean, Phys. Rev. 102, 1413 (1956).

[27] R. Skomski, H. P. Oepen, and J. Kirschner, Phys. Rev. B 58, 3223 (1998).

[28] V. I. Nikitenko et al., Phys. Rev. B 57, R8111 (1998).

[29] V. I. Nikitenko et al., Phys. Rev. Lett. 84, 765 (2000).

[30] A. Kubetzka et al., Phys. Rev. Lett. 94, 087204 (2005).

[31] A. A. Belavin and A. M. Polyakov, Pis'ma Zh. Eksp. Teor. Fiz. 22, 503 (1975) [JETP Lett. 22, 245 (1975)].

[32] A. Abanov and V. L. Pokrovsky, Phys. Rev. B 58, R8889 (1998). 